Research Notes

\title{
A Comprehensive Study on Mobility Issues in AODV
}

\author{
Thiripurasundari Sivakrishnakumar \\ Department of Computer Science and Engineering, Rajiv Gandhi College of Engineering, Chennai, India
}

Article history

Received: 07-08-2015

Revised: 01-12-2015

Accepted: 14-12-2015

Email: thiripurasundaricse27@gmail.com

\section{Introduction}

Objective of a network forum is to enable all the connected IoT to participate and share the information irrespective of where they are. Wireless networks are special kind of networks which consists of mobile terminals, connect through either radio waves or micro waves, by peer to peer communication basis. Ad hoc networks are non-infrastructure networks as they don't have fixed access point and it exists for a short duration of time. By means of co-operative multi hop technique; these nodes can propagate messages even beyond their communication range. The importance of MANET in internet proves to be dominant in most of the recent technologies includes cloud computing. Routing is the process by which forwarding tables are built on each node periodically, according to those individual packets are routed and delivered to the corresponding site. As mobile nodes are randomly moving, the route discovery and maintenance procedures are energy consuming on this battery driven terminals. Once the path breaks, data packets will be lost or delayed for some time, until the reconstruction of route is done. Applications of these independent ad hoc networks include armed forces, emergency disaster management and under mining operations.

Dynamic routing protocols are suited to MANET, due to the changes in the network topology over time and they are classified into two categories: Proactive and
Reactive. Proactive routing protocols are based on prior building of routing tables and to forward the packets and updating their routing table continuously (e.g.,) Distance Sequenced Distance Vector (DSDV). When there is a need, then the building process of routing table will be performed on the involved nodes. This category of protocols belongs to reactive protocols (e.g.,) AODV and Temporally Ordered Routing Algorithm (TORA). In real world scenarios, reactive protocols proved better performance in MANET (Pirzada et al., 2006), in which we have chosen standard AODV routing protocol (Perkins et al., 2003) inspired by RFC 3561, for this performance assessment. Although there are many papers available that examines the performance of AODV using various simulators like NS-2, here the experiments are carried out related to real time scenario and considered all possible parameters that figure out AODV. The interesting feature of AODV is, it is quick response towards dynamic route changes compared with other protocols. It supports unicast and can be extensible to multicast and any-cast communication. It uses sequence number field in its packet header to identify routing loops and latest route updates (Hallani and Shahrestani, 2008). In this study, evaluating AODV's behavior is done with respect to Wi-Fi nodes' mobility in sparse and dense networks using NS-3 simulator. NS3 is a recent emerging open source simulator providing platform to assess the performance of wireless networks (Honderson, 2009; Ikeda et al., 2011). 
Rest of the paper is arranged as follows. Section II reviews the literature. Simulation setup and numerical results of AODV's performance is presented in section III. Finally, the paper is concluded in section IV.

\section{Literature Review}

As per Wireless Broadcast Average (WBA) principal, nodes inside transmission range of another node may receive the packet; they serve as co-operative caching and backup nodes if the intended receiver fails to receive the packet (Huang et al., 2008). Biggest issue in MANET research is routing; with rapid and diverse nature of growing mobile nodes, the choice of routing protocol is likely to determine the quality of application requirements (Toh et al., 2002). Due to node mobility, links become unavailable; it leads to a situation which triggers path recovery mechanism in unpredictable manner. Fluctuations of link and path durations are expected to shape the performance and overheads of the on-demand routing protocols. AODV is a popular reactive protocol and it can cope with mobility induced routing failures even bandwidth of wireless channel is limited. AODV is utilizing the route cache on mobile nodes efficiently unlike other reactive protocols and easy to deployable in unmanned distributed surveillance and tracking operations (Valera et al., 2005).

Opportunistic MANETs are special class of disconnected sparse network where flooding time is a decreasing function of the maximal speed information is fast, because of the node's mobility. In recent years, this special kind of MANET is getting important as it provides opportunities for energy saving and other parameters (Wieselthier et al., 2001). Node's mobility is an important factor to assess the characteristics of the routing protocol performance (Yang et al., 2012). Flowmonitor is an add-on module which is implemented in NS-3 (Carneiro et al., 2009). Flowmonitor is used to separate dataflow and control flow in AODV routing protocol and used to filter per flow based on our interest at each node. In this study, AODV is modeled and assessed its performance by means of computing aggregated throughput, end to end delay and packet loss ratio. Here, the aim is to capture AODV's scalability with respect to mobile nodes' mobility in sparse network and dense network scenario as well.

\section{Results}

\section{Simulation Setup}

The primary purpose of investigation is to develop an understanding and hands on appreciation of real time behavior of AODV inspired by Agent Based
Model (ABM). Here, the scenario considered is a uniformly distributed wireless network which is imitating the real world situation where the nodes are definite and spread over the region, with reference to disaster recovery management. This AODV routing protocol is built on the top of standard IEEE 802.11 MAC layer where node's mobility model is set to Random Waypoint (RWP) (Yoon et al., 2003). Nodes' mobility start up time is set as zero pause time, so that the Wi-Fi nodes can collect less information from the network in order to setup a typical Ad-hoc environment (Marina and Das, 2006). Each node maintains a routing table updated during the route-discovery of protocol. The following Fig. 1 gives the snapshot of sparse network model created in NS-3 simulator. It shows 10 number of Wi-Fi nodes which are randomly distributed over $800 \mathrm{~m} \times 700 \mathrm{~m}$ rectangle region.

Sparse and dense network scenarios are simulated using NS-3 simulator, in which all nodes are operating in AODV for routing. Here, the assumption is that nodes are doing secure communication and no node going to break down during this period. Total simulation duration is set to $800 \mathrm{~s}$, so that all kinds of uncertainties are taken into account. A maximum speed of around $20-50 \mathrm{~m} / \mathrm{s}$ is set as node speed, which was followed in the most of the survey papers (Paul et al., 2000). For each node, a transmission range is considered an area in which the strength of the emitted signals from the node is strong enough to be sensed by other nodes. It depends on power and models unidirectional connectivity nodes.

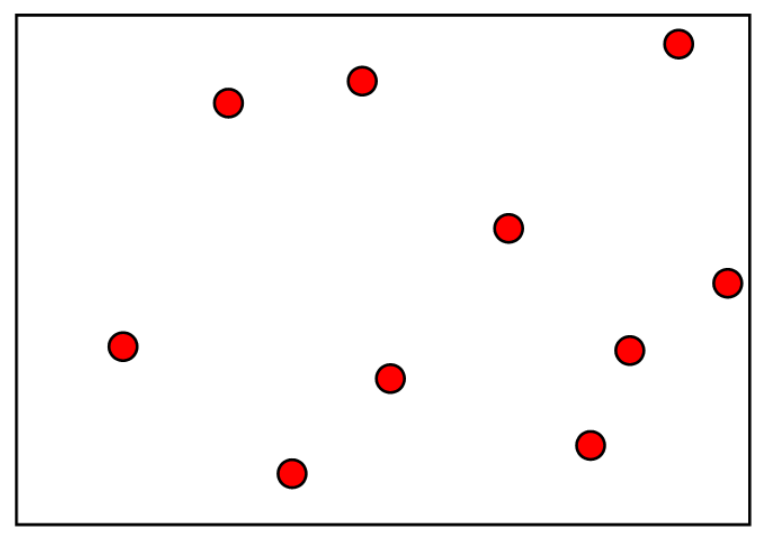

Fig. 1. Network model

Table 1. Simulation parameters

\begin{tabular}{ll}
\hline & 10 nodes for sparse network and \\
Number of nodes & 30 nodes for dense network scenario \\
\hline Node type & Wi-fi $802.11 \mathrm{~b}$ radios \\
Transmission power & $8.9048 \mathrm{dBm}$ \\
Node speed & Range from $5 \mathrm{~m} / \mathrm{s}$ up to $25 \mathrm{~m} / \mathrm{s}$ with \\
& zero pause time \\
Traffic pattern & UDP On/Off application on CBR \\
\hline
\end{tabular}


Nodes' transmission ranges are elaborated up to $250 \mathrm{~m}$ as in (Han et al., 2006) where all nodes are moving according to RWP. Rest of the simulation parameters were chosen based on existing MANET experiment related materials (Ghassemi et al., 2010). If two nodes are in same region, the emitted signals may interfere during broadcasting and it will be handled by IEEE 802.11 MAC protocol to reduce the interference; but not to recovery the lost data. The following Table 1 shows the simulation setup.

\section{Simulation Results and Discussion}

With reference to Table 1, simulation is done among randomly positioned $10 \mathrm{Wi}-\mathrm{Fi}$ nodes where nodes' mobility speeds are varied from 5 to $25 \mathrm{~m} / \mathrm{s}$. The derived QoS parameters are calculated out of 10 numbers of simulations. Five number of source/sink pairs are used to generate concurrent UDP On/Off application in CBR in sparse network scenario. Ten number of source/sink pairs are used to generate concurrent UDP On/Off application CBR data communication in dense network scenario. QoS parameters are computed with respect to node's mobility value where the transmission power is same from the starting of simulation to the end. The following graphs are generated as the result from observation. Figure 2 shows the throughput obtained at each mobile speed intervals. Here, the aggregated throughput is computed with respect to the number of bits successfully received with unit time.

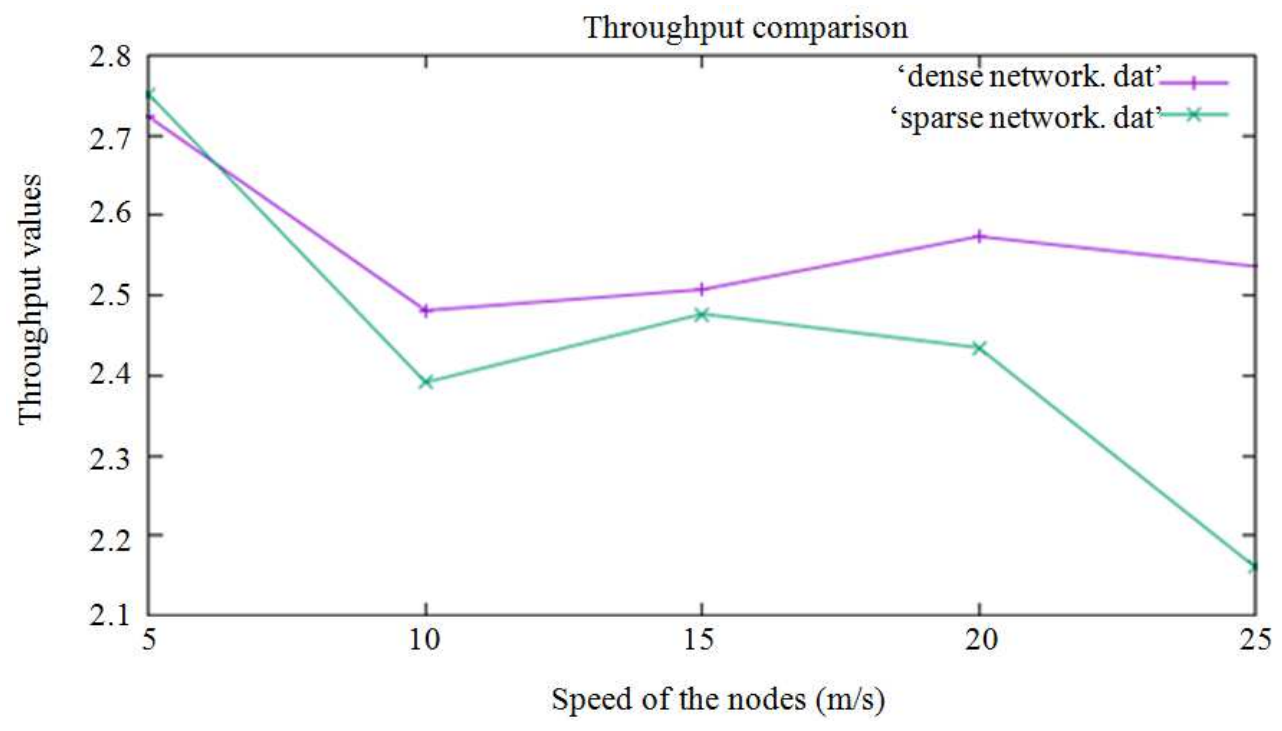

Fig. 2. Throughput distribution

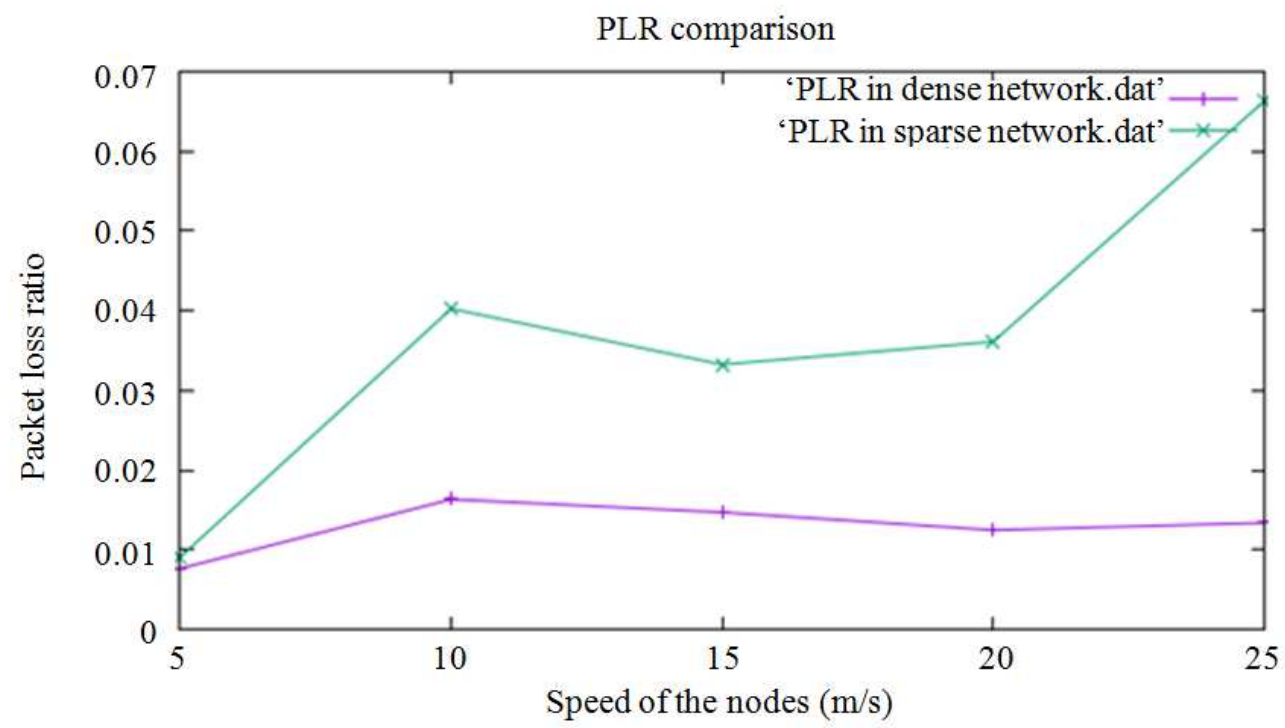

Fig. 3. End to end delay distribution 


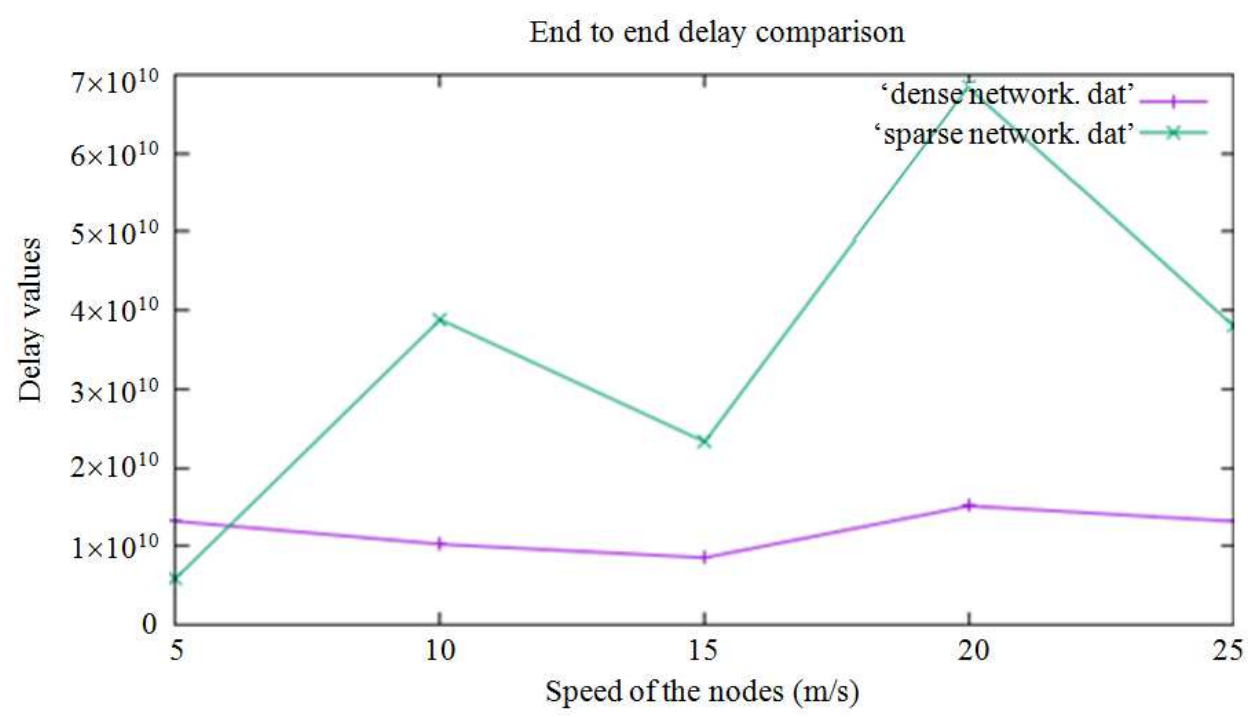

Fig. 4. PLR distribution

Average end-to-end delay is considered as the sum of the delay of all received packets divided by the number of received packets. The following Fig. 3 shows the end to end delay experienced by nodes in sparse and dense networks as well. This end-to-end delay parameter of AODV protocol seems to be poor performance in sparse network scenario and survival of MANET become poor in this worst case.

The following graph in Fig. 4 shows the PLR distribution ranges over the given range of mobile node speed. PLR is computed as the ratio of lost packets to the sum of all received and lost packets. As the mobile node's speed increases, PLR is also increasing gradually. It is observed that PLR ratio is higher in sparse network scenario than dense network scenario. This better result is due to the availability of mobile nodes in dense network, which in turn become the advantage for reconstructing the routes quickly.

\section{Conclusion}

Mobility of the nodes causes unpredictability in the wireless links of MANET. Here the belief is that the results presented in this study provide a better understanding of AODV routing behavior with respect to mobility, particularly where nodes are in random motion. It is showed through extensive simulations which are aggregated the necessary parameters in radio networks by simulations. From the results, AODV is identified as it performs well in the dense network scenario, when the node's speed is within small range and provided reliable packet delivery. When the degree of the mobility is increased, standard AODV routing protocol differs from expected packet delivery and its performance gradually decreased especially in sparse network. Extending this work to compute other related
QoS performance attributes is the future work. Consideration of changing the default values of AODV may be tuned to get better results.

\section{Acknowledgement}

My sincere thanks to Prof. Dr. T.S. Jeyali Laseetha and Mrs. Sujitha AP/CSE for their constant support.

\section{Author Contribution}

Done data gathering and simulation of AODV in NS3. Compilation of my research paper.

\section{Ethics}

This article is original and contains unpublished material. The corresponding author confirms that all of the other authors have read and approved the manuscript and no ethical issues involved.

\section{References}

Carneiro, G., P. Fortuna and M. Ricardo, 2009. FlowMonitor: A network monitoring framework for the Network Simulator 3 (NS-3). ICST, Pisa, Italy.

Ghassemi, F., W. Fokkink and A. Movaghar, 2010. Equational reasoning on $\mathrm{Ad}$ Hoc networks. Proceedings of the 3rd IPM International Conference on Fundamentals Software Engineering, Apr. 15-17, Springer, Kish Island, pp: 113-128. DOI: 10.1007/978-3-642-11623-0_6

Hallani, H. and S.A. Shahrestani, 2008. Improving the reliability of ad-hoc on demand distance vector protocol. WSEAS Trans. Commun., 7: 695-704. 
Han, Y., R.J. La, A.M. Makowski and S. Lee, 2006. Distribution of path durations in mobile Ad-hoc networks-Palm's theorem to the rescue. Comput. Netw., 50: 1887-1900.

DOI: 10.1016/j.comnet.2005.10.005

Honderson, T., 2009. NS-3 tutorial. SIMUTools.

Huang, X., H. Zhai and Y. Fang, 2008. Robust cooperative routing protocol in mobile wireless sensor networks. IEEE Trans. Wireless Commun., 7: 5278-5285. DOI: 10.1109/T-WC.2008.060680

Ikeda, M., M. Hiyama, E. Kulla and L. Barolli, 2011. Mobile ad-hoc network routing protocols performance evaluation using NS-3 simulator. Proceedings of the 3rd International Conference on Intelligent Networking and Collaborative Systems, Nov. 30-Dec. 2, IEEE Xplore Press, Fukuoka, pp: 14-20. DOI: 10.1109/INCoS.2011.119

Marina, M.K. and S.R. Das, 2006. Ad hoc on-demand multipath distance vector routing. Wireless Commun. Mobile Comput., 6: 969-988.

DOI: $10.1002 / \mathrm{wcm} .432$

Paul, K., S. Bandyopadhyay and R. Roychoudhuri, 2000. Survivable Ad hoc wireless networks: Some design specifications. Multiaccess, Mobility Teletraffic Wireless Commun., 5: 147-158. DOI: 10.1007/978-1-4757-5916-7_13

Perkins, C., E. Belding-Royer and S. Das, 2003. Ad hoc On-demand Distance Vector (AODV) routing. RFC.
Pirzada, A.A., C. McDonald and A. Datta, 2006. Performance comparison of trust-based reactive routing protocols. IEEE Trans. Mobile Comput., 5: 695-710. DOI: 10.1109/TMC.2006.83

Toh, C.K., M. Delwar and D. Allen, 2002. Evaluating the communication performance of an $\mathrm{Ad}$ hoc wireless networks. IEEE Trans. Wireless Commun., 1: 402-414. DOI: 10.1109/TWC.2002.800539

Valera, A.C., W.K.G., Seah and S.V. Rao, 2005. Improving protocol robustness in Ad hoc networks through cooperative packet caching and shortest multipath routing. IEEE Trans. Mobile Comput., 4: 443-457. DOI: 10.1109/TMC.2005.67

Wieselthier, J.E., G.D. Nguyen and A. Ephremides, 2001. Algorithms for energy-efficient multicasting in static Ad hoc wireless networks. Mobile Netw. Appli., 6: 251-263. DOI: $10.1023 / \mathrm{A}: 1011478717164$

Yang, S., C.K. Yeo and B.S. Lee, 2012. Toward reliable data delivery for highly dynamic mobile Ad hoc networks. IEEE Trans. Mobile Comput., 11: 111-124. DOI: 10.1109/TMC.2011.55

Yoon, J., M. Liu and B. Noble, 2003. Random waypoint considered harmful. Proceedings of the 22nd Annual Joint Conference of the IEEE Computer and Communications IEEE Societies INFOCOM, Mar. 30-Apr. 3, IEEE Xplore Press, San Francisco, CA., pp: 1312-1321.

DOI: 10.1109/INFCOM.2003.1208967 